\title{
A PHOTOMETRIC ATTACHMENT FOR SPECTROSCOPES
}

\author{
By P. G. Nutting
}

The instrument here described was designed for spectrophotometric work and with three ends in view: (I) High sensibility, (2) economy of light, and (3) variable dispersion. Tests of a sample instrument constructed by Fuess show that the sensibility has been pushed to the limit of sensibility ( 1.5 per cent) of

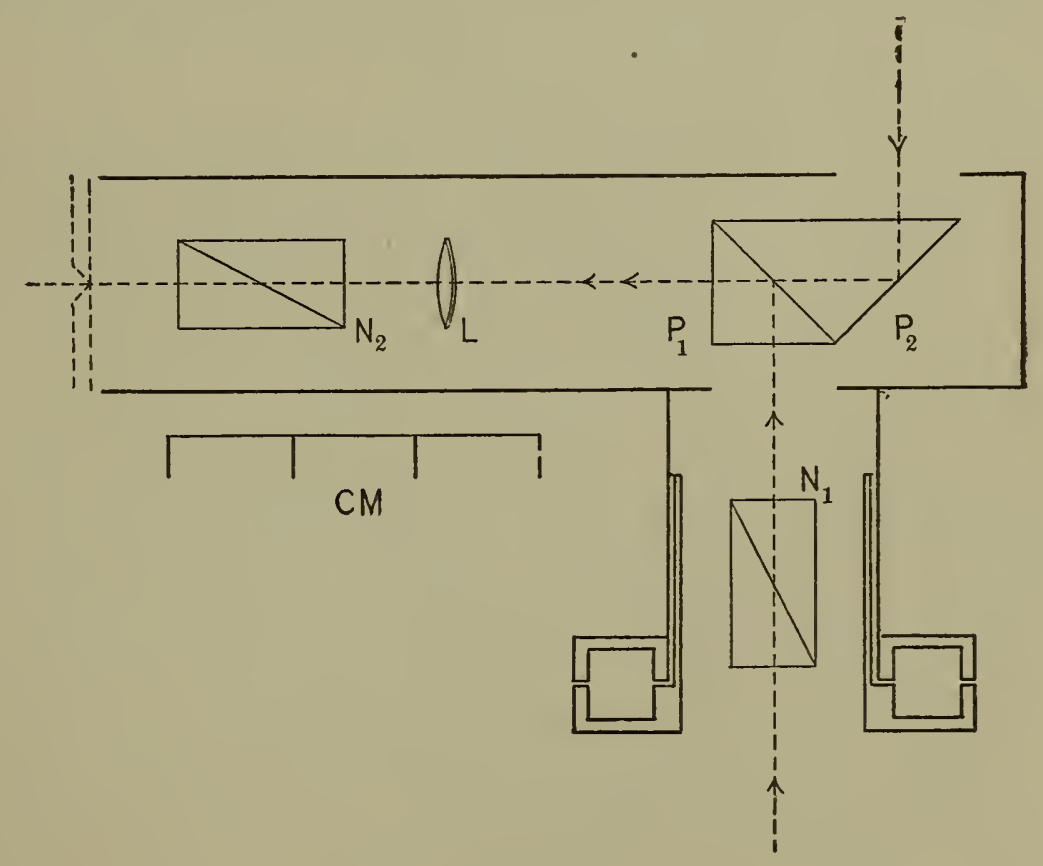

Fig. 1

the eye, while the light economy is quite satisfactory and the dispersion that of any spectroscope to which it is attached. No trace of stray light can be detected in the new instrument and the single face on which dirt could cause stray light is entirely inclosed. There is a slight correction to the scale reading, but this is less than 2 per cent at its maximum. 
The attachment consists of two reflecting prisms, $\mathrm{P}_{1}$ and $\mathrm{P}_{2}$, two Glan-Thompson nicols $\mathrm{N}_{1}$ and $\mathrm{N}_{2}$ and a lens arranged as shown in the figure. The whole is attached to the slit of any spectroscope, for example one of the Hilger "wave length" type. The essential feature is that a real image of the photometric surface (the common surface of the two reflecting prisms) is thrown on the slit by an achromatic lens and may thus be brought into the plane of the slit. The two beams of light to be compared, one passing through and the other reflected from the photometric surface, are brought to equality by rotating the nicol $\mathrm{N}_{1}$. The photometric surface is alternately silvered and clear, the silver strips being $0.25 \mathrm{~mm}$ wide and separated by clear strips equal in width to the silvered strips.

The combination of reflecting prisms was suggested by and is similar to that used by Dr. H. E. Ives ${ }^{1}$ in his new spectrophotometer, but in his instrument the photometric surface is silvered halfway across instead of in fine strips.

Both beams are polarized by reflection and the instrument was carefully tested for departures from the cosine square law of intensities. First the reflecting system was removed and the elliptical polarization (change of phase and rotation) produced by reflection on each of the reflecting surfaces determined for $45^{\circ}$ incidence and $45^{\circ}$ azimuth. The second test was by means of an auxiliary nicol placed before the free opening so that both beams were polarized before reflection. The third test was with rotating sectored disks of fixed known openings mounted before the free opening of the instrument. In this last test the sources were seasoned glow lamps operated in series with a rheostat on a storage battery circuit. The distances of these lamps were adjusted to equality of illumination with the $180^{\circ}$ disk moving and the nicol at $0^{\circ}$. Then other sectors were substituted for the $180^{\circ}$ sector and the readings of the nicol compared with their theoretical values deduced from the sector openings. The corrections were nowhere in excess of 2 per cent and of such sign as to indicate that they were in large part due to accidental errors.

The sensibility of the instrument was tested by rotating a parallel glass plate of known index in the path of one beam and

${ }^{1}$ Phys. Rev., 30, p. 450; I9ro. 
by repeating readings. Sensibilities varying from 0.015 to o.or 8 were recorded.

The excellent showing made by the instrument is due in some measure to the excellent workmanship displayed in the construction of its optical parts. But two minor improvements suggest themselves; that the position of the lens be made adjustable from without and that the balancing nicol $\mathrm{N}_{1}$ be provided with a larger (ro $\mathrm{cm}$ ) divided circle to permit of more precise readings.

WASHINGTON, October I, I9IO. 